\title{
Cytolytic effects and apoptosis induction of newcastle disease virus strain AF2240 on anaplastic astrocytoma brain tumor cell line.
}

\begin{abstract}
Newcastle disease virus (NDV) is a member of genus Avulavirus within the family Paramyxoviridae. Interest of using NDV as an anticancer agent has arisen from its ability to kill tumor cells with limited toxicity to normal cells. In this investigation, the cytotolytic properties of NDV strain AF2240 were evaluated on brain tumor cell line, anaplastic astrocytoma (U-87MG), by using MTT assay. Cytological observations were studied using fluorescence microscopy and transmission electron microscopy to show the apoptogenic features of NDV on U-87MG. DNA laddering in agarose gel electrophoresis and terminal deoxyribonucleotide transferase-mediated dUTP-X nick end-labeling staining assay confirmed that the mode of cell death was by apoptosis. However, analysis of the cellular DNA content by flowcytometery showed that there was a loss of treated U-87MG cells in all cell cycle phases (G1, S and G2/M) accompanied with increasing in sub-G1 region (apoptosis peak). Early apoptosis was observed $6 \mathrm{~h}$ post-inoculation by annexin-V flow-cytometry method. It could be concluded that NDV strain AF2240 is a potent antitumor agent that induce apoptosis and its cytotoxicity increasing while increasing of time and virus titer.
\end{abstract}

Keyword: Newcastle disease virus; Apoptosis; Flow-cytometry; Cytotoxicity; Anaplastic astrocytoma. 